\title{
SIGNAL: SAR FOR ICE, GLACIER AND GLOBAL DYNAMICS
}

\author{
Thomas Börner ${ }^{1}$, Francesco De Zan ${ }^{1}$, Paco López-Dekker ${ }^{1}$, Gerhard Krieger ${ }^{1}$, Irena Hajnsek ${ }^{1}$, Kostas \\ Papathanassiou $^{1}$, Michelangelo Villano ${ }^{1}$, Marwan Younis ${ }^{1}$, Andreas Danklmayer ${ }^{1}$, Wolfgang Dierking ${ }^{2}$, \\ Thomas Nagler ${ }^{3}$, Helmut Rott ${ }^{3}$, Susanne Lehner ${ }^{4}$, Thomas Fügen ${ }^{5}$ and Alberto Moreira ${ }^{1}$
}

(1) German Aerospace Center (DLR), Microwaves and Radar Institute

(2) Alfred-Wegener-Institut

(3) University of Innsbruck, Institute of Meteorology and Geophysics

(4) German Aerospace Center (DLR), Remote Sensing Technology Institute

(5) EADS Astrium GmbH

\begin{abstract}
SIGNAL is an innovative earth exploration mission proposal with the main objective to estimate accurately and repeatedly topography and topographic changes associated with mass change or other dynamic effects on glaciers, ice caps and polar ice sheets. Elevation measurements are complemented with glacier velocity measurements, providing valuable additional information for a better understanding of the hydrology of glacierized basins and of the Arctic and Antarctic water cycle. SIGNAL is capable of monitoring all critical regions with a high spatial resolution and an adequate revisit time. This paper gives an overview about the actual mission design status and provides a brief description of the topography (DEM - digital elevation map) selfcalibration strategy and the estimated global interferometric performance.
\end{abstract}

Index Terms — SAR, Mission, Ice, Glacier, Ka-band

\section{INTRODUCTION}

Recent observations indicate a dramatic increase of ice mass losses from glaciers, ice caps, and the Greenland and Antarctic ice sheets [1]. As a result, several new studies predict a global mean sea level rise more than twice as large as the projections from the IPCC 2007 report [2]. From observations, delivered by satellites and conventional observing systems, it is obvious that the Cryosphere reacts very sensitively to climate change. However, the feedbacks to the global climate system are not well understood, impairing predictions of the impact of future climate change. Improved observational data are needed to better quantify the main cryospheric processes and improve the representation of the Cryosphere in climate models. One key parameter that is missing for a better understanding of the intricate dynamics of ice mass balances is a detailed and accurate knowledge of the 3-D ice surface topography and its changes at fine spatial and temporal resolutions.
The main objective of SIGNAL is to fill major gaps in the data base on mass balance and dynamics of global glacier ice and to thus advance in the knowledge of the processes governing the response of the ice masses to climate forcing. The mission addresses those components of the ice budget that have been subject to accelerated downwasting during the last decade and for which the knowledge of the present mass balance and temporal trends exhibits large error bars: the mountain glaciers and ice caps, and the outlet glaciers of the boundary zones of the Greenland and Antarctic ice sheets. There is no mission in space or in preparation that is particularly devoted to observing the mass changes of these components of the global Cryosphere at high spatial detail.

The primary mission objectives are thus defined as:

- Reducing the uncertainty in the mass balance of glaciers and ice caps;

- Improving the knowledge on mass depletion of outlet glaciers in Greenland and Antarctica.

The secondary mission objectives are:

- Downscaling of altimetric elevation data over ice sheets;

- Mapping the motion of calving glaciers and ice streams in support of mass balance retrieval;

- Supporting the protection from natural hazards related to major mass movements;

- Assessing the new opportunities of high-frequency, high resolution interferometric SAR data for sea ice surface parameter retrieval.

In order to meet these objectives repeat measurements of surface elevation of the global glaciers and ice caps are aspired. Accurate and consolidated numbers on the global change in glacier mass can be achieved by performing repeat measurements of the global glacier volume in seasonal and annual time intervals. To establish and calibrate models of mass balance and atmospheric forcing, the seasonal measurement of volume changes for a representative subsample of glaciers around the world is proposed. The meas- 
urements of glacier motion will focus on calving glaciers and on outlet glaciers of ice sheets, in order to retrieve the ice export. This is needed to determine the mass balance for these glacier types, in addition to volume changes.

\section{MISSION OUTLINE}

The main driver for the mission design is the generation of digital elevation models of all relevant areas with height accuracies in the order of a few decimetres. This goal justifies two fundamental choices:

- The use of Ka-band (35 GHz) to minimize the penetration into the ice or snow cover, in order to obtain a DEM that is truly representative of the surface.

- The use of a pair of formation flying satellites. This is the only way to obtain the long base-lines, roughly $100 \mathrm{~m}$, required to achieve the desired height sensitivity and measurement stability, avoiding temporal decorrelation effects.

SIGNAL is designed to obtain sub-meter height accuracies that range from $10 \mathrm{~cm}$ to $1 \mathrm{~m}$, depending on the application and the required product resolution. The system will benefit from the possibility of using a very large number of looks resulting from the combination of moderate product resolution requirements (50 to $200 \mathrm{~m}$ ) with a high resolution SAR system. The convergent satellite tracks near the northern and southern turns also provide a unique opportunity for innovative calibration techniques.

SIGNAL is planned as a systematic mapping mission with a lifetime of at least 5 years that will generate seasonal DEMs of the areas of interest. In addition to its mission driving interferometric capabilities, SIGNAL will also be a very capable system to estimate glacier velocities using incoherent feature tracking techniques [3]. These techniques are of particular interest for fast-flowing glaciers, for which coherent techniques fail due to temporal decorrelation.

To optimally address the main topographic mission requirements but also the secondary glacier velocity measurements, the mission will be divided into three recurring scientific phases:

1. A DEM acquisition phase aimed at obtaining an initial DEM.

2. A DEM tracking phase aimed at monitoring DEM variations with very high accuracy.

3. A glacier velocity monitoring phase. During this, one of the spacecrafts will be rotated with respect to the other, thereby doubling the spatial coverage.

The baseline design consists of two identical satellites, each featuring a Ka-band SAR system with a $2.1 \mathrm{~m}$ x $1.3 \mathrm{~m}$ reflector antenna. The radar will use highly innovative digital beamforming techniques [4] to achieve the minimum required $20 \mathrm{~km}$ swath from a $740 \mathrm{~km}$ sun-synchronous orbit with a repeat period of 11 days.

Complete coverage of the target areas will be achieved in 2 months using an appropriate attitude steering scheme. A formation flying concept similar to that of TanDEM-X will be used, but in a pursuit monostatic configuration, which is easier to operate and reduces risks and costs.

\section{DEM SELF-CALIBRATION CONCEPT}

Aside from noise-like phase errors, which can be mitigated by averaging independent looks, the overall performance of SIGNAL is limited by low frequency systematic phase offsets and baseline uncertainties. In most InSAR scenarios, systematic errors are dealt with by tying the results to reference ground control points. In that sense, InSAR is understood as a technique that is good at measuring relative topographic variations that are usually local phenomena. SIGNAL is different, because it aims at monitoring height variations over large extensions in areas, where the density of ground control points may be low. The main goal of this section is to show that, in SIGNAL, systematic errors can be dealt with even in the absence of stable control points, introducing a self-calibration approach. This self-calibration exploits three characteristics of the proposed mission:

1. Baseline and phase offset uncertainties can be modeled as smooth random processes that can be characterized in terms of autocorrelation functions or covariance matrices.

2. There is a lot of redundancy in the data due to overlapping swathes. In particular, at high latitudes, there are a lot of points that are covered by crossing, ascending and descending, data-takes.

3 . The systematic phase errors and the possible topography variations have distinct spatio-temporal spectral components and can, therefore, be jointly estimated.

\subsection{Baseline errors as stochastic process}

The relative baseline between the two antennas will be determined with high precision from double difference GPS measurements. However, there will remain errors in the millimeter range that will still affect the DEM products. An error in the determination of the baseline will correspond to a phase error across the swath. When this phase error is converted to height (according to the phase-to-height coefficient) it will result into a height error. The baseline error is modeled as an autoregressive process with two poles (in the z-transform), symmetric with respect to the real axis. The closer the poles are to the unitary circle, the more coherent the process is over time. The phase of the poles corresponds to the orbital period, as observed and derived from 
very accurate along-track baseline measurements from the GRACE mission [5].

\subsection{Calibration with crossing points}

When an area is imaged by two orbits (one ascending and one descending, see fig. 1) the opportunity for calibration is given. The slopes induced by the two baseline errors are mostly uncorrelated and - more important - they have different characteristics. In particular the error vanishes at different points, since the two orbits will have different nadir projections on the ground. The problem would be easy if there were no additional errors. However, in the first place, interferometric measurements are not error free: the compared height will have errors due to coherence loss and other factors. Second, the height itself could have changed between the two data takes, i.e. a physical height error could be mistaken with a baseline-induced error.

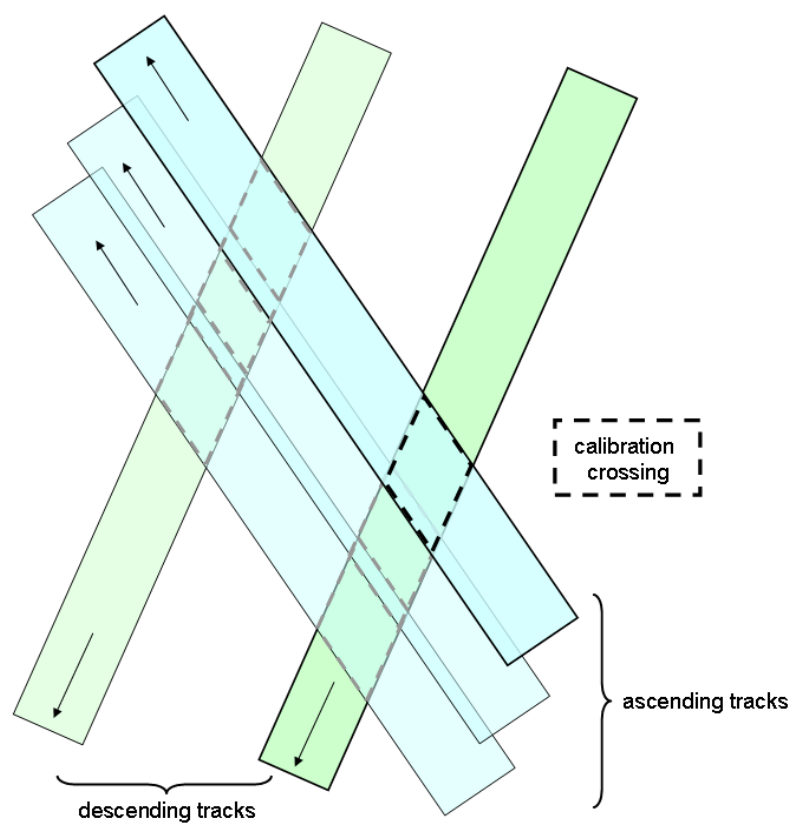

Figure 1: Geometry of track crossings for calibration.

For every orbital crossing an equation like the following can be written:

$$
\tilde{z}=k \cdot g r+z_{0}+z(t)
$$

The term $z_{0}$ represents the reference height, $k$ the baselineerror induced slope and $g r$ the ground range coordinate. The time-varying term $z(t)$ accounts for both physical height change and interferometric noise (SNR, ambiguities, etc.). When taking the differences between the heights measured from two different passes, the mean value of the height $z_{0}$ cancels out and is no more relevant. The time varying part $z(t)$ will not, since some time will elapse between the passes. The slope-error $k$ will also contribute to the measured height difference.

$$
\Delta \tilde{z}=k\left(t_{1}\right) g r_{1}-k\left(t_{0}\right) g r_{0}+\Delta z\left(t_{0}, t_{1}\right)
$$

Here $g r_{1}$ and $g r_{0}$ represent the ground-range coordinates of the measured point from the two different passes. The slopes $k$ are represented as a function of time. Each calibration point yields one such equation and thus can be collected in a matrix form as:

$$
\Delta \tilde{\mathbf{z}}=\mathbf{M k}+\Delta \mathbf{z}
$$

\subsection{Slope estimation and performance}

The Linear Minimum Mean Square Error (LMMSE) estimator of vector $\mathbf{k}$ is an optimal linear combination of the observations $\boldsymbol{\Delta} \tilde{\mathbf{z}}$, i.e.

$$
\hat{\mathbf{k}}=\mathbf{A} \Delta \tilde{\mathbf{z}},
$$

where the matrix $\mathbf{A}$ is built so that the mean square error is minimized in a statistical sense. For the purpose of deriving the performance it is not necessary to write the explicit expression for $\mathbf{A}$, because the final performance is provided by the a posteriori covariance matrix $\mathbf{R}_{\hat{k}}$ of the vector $\hat{\mathbf{k}}$, which can be written as [6]:

$$
\mathbf{R}_{\hat{k}}=\left[\mathbf{R}_{k}^{-1}+\mathbf{M}^{T} \mathbf{R}_{z}^{-1} \mathbf{M}\right]^{-1}
$$

This expression can be interpreted saying that the a posteriori information on the baseline error (the ground-range slope) is equal to the a priori information $\mathbf{R}_{\mathrm{k}}$ (derived from the autoregressive model) plus the noise information projected onto the baseline error space.

The performance of the DEM calibration depends on the error of the baseline and on the characterization of the correlations of height errors at different points and different times. By modeling these error sources for a given scenario of crossings over Greenland (see fig. 2) it is possible to derive the a posteriori covariance of the slopes. By multiplying these residual slope errors with the ground range distance (e.g. far ground range of the $20 \mathrm{~km}$ swath for worst case), we derive the residual height error for a DEM measurement over Greenland. The result for three different baselines is presented in fig. 3 and shows that the baseline does not have a big impact on the calibration and that we can expect a worst case height error around $10-15 \mathrm{~cm}$. Since the number of crossings increases with latitude, the performance will, in general, be better for higher latitudes.

\section{GLOBAL INTERFEROMETRIC PERFORMANCE}

The global interferometric error budget includes contributions from noise like errors and systematic errors and should consider also all the data acquired during the entire mission. This mission wide analysis is, however, beyond the scope of this proposal, in part because it is foreseen that the availability of the data set produced by SIGNAL will lead to the development of improved models and inversion algorithms. However, a conservative estimation of the global perform- 
ance can be given by the single DEM accuracy and by the acquisition to acquisition error budget.

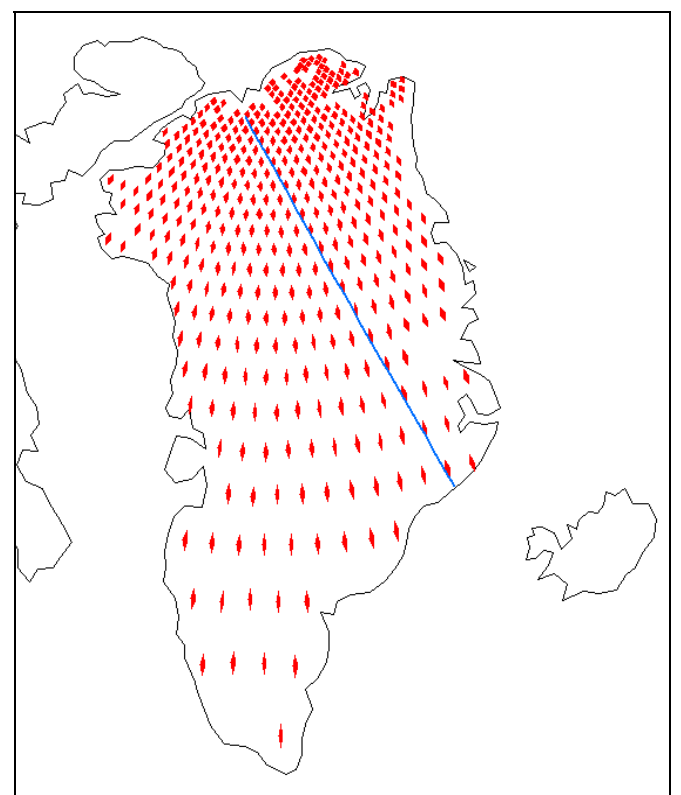

Figure 2: Crossing areas of ascending and descending $20 \mathrm{~km}$ swathes (red) over Greenland for an 11 day repeat period and the ground track of an arbitrarily chosen orbit (blue).

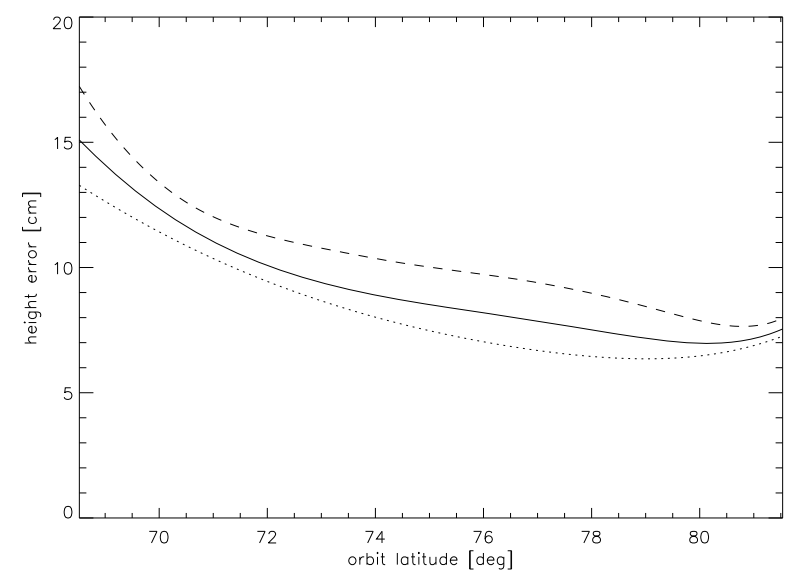

Figure 3: Residual height error due to baseline errors for a flight over Greenland, calibrating using all possible crossings over Greenland in a 11 day repeat cycle, for monostatic baselines of 50 m (dashed line), $100 \mathrm{~m}$ (solid line) and $200 \mathrm{~m}$ (dotted line).

A summary of the performance estimates is given in table 1 , where the applications and product resolutions refer to the science requirements. The fourth and fifth columns give the $90 \%$ confidence height errors, while the sixth and seventh columns reproduce the minimum and target science requirements, which here are interpreted as $90 \%$ confidence intervals. There requirements have been color-coded, with green indicating that the requirement is completely or nearly satisfied, yellow indicating that it is partially met, and orange indicating that is not met.

\begin{tabular}{|c|c|c|c|c|c|}
\hline \multirow{2}{*}{ Application } & \multirow{2}{*}{$\begin{array}{c}\text { Product } \\
\text { Res. [m] }\end{array}$} & \multirow{2}{*}{$\begin{array}{c}\text { hamb } \\
{[\mathbf{m}]}\end{array}$} & $\begin{array}{c}\text { Predicted } \\
\text { Height } \\
\text { Error [m] }\end{array}$ & \multicolumn{2}{|c|}{$\begin{array}{c}\text { Science } \\
\text { Req. [m] }\end{array}$} \\
\cline { 5 - 7 } & & $\mathbf{M}$ & $\mathbf{T}$ \\
\hline Ice dynamic & 100 & 14 & $0.37-0.44$ & 1.0 & 0.5 \\
\hline Mass balance & $200(100)$ & 14 & $0.35-0.37$ & 0.5 & 0.2 \\
\hline \multirow{2}{\text{Icesheets}}{\begin{tabular}{c} 
DEM \\
\cline { 5 - 7 }
\end{tabular}} & $100(50)$ & 14 & $0.11-0.27$ & 0.3 & 0.1 \\
\cline { 5 - 7 } & 100 & 14 & $0.29-0.49$ & 0.3 & 0.1 \\
\hline Hazards & 50 & $14-20$ & $0.41-0.63$ & 2 & 0.5 \\
\hline
\end{tabular}

Table 1: Global interferometric performance. Errors are $90 \%$ confidence values. (M-Minimum, $T$ - Target)

A first performance assessment of the feature-tracking capabilities of SIGNAL based on TerraSAR-X data over glaciers has been carried out and published in [6].

\section{ACKNOWLEDGEMENTS}

The SIGNAL project is supported by the German Space Agency of the German Aerospace Center (DLR) with funds of the Federal Ministry of Economics and Technology (BMWi) under support code 50 EE 0931.

\section{REFERENCES}

[1] H. D. Pritchard, R. J. Arthern, D. G. Vaughan, and L. A. Edwards, "Extensive dynamic thinning on the margins of the Greenland and Antarctic ice sheets," Nature, doi:10.1038/nature08471.

[2] IPCC Fourth Assessment Report: Climate Change 2007 - Synthesis Review.

[3] N.H. Short, and A.L. Gray, "Potential for RADAR-SAT-2 interferometry: glacier monitoring using speckle tracking," Can. J. Remote Sensing, vol. 30, no. 3, pp. 504-509, June 2004.

[4] G. Krieger, N. Gebert, M. Younis, F. Bordoni, A. Patyuchenko, and A. Moreira, "Advanced concepts for ultra-wide swath SAR imaging,” Proc. European Conference on Synthetic Aperture Radar EUSAR 2008, Friedrichshafen, Germany, June 2008.

[5] J. Hueso Gonzáles et al., "Development of the TanDEM-X Calibration Concept: Analysis of Systematic Errors”, IEEE Trans. Geosci. Remote Sens., vol. 48 (2), 2010.

[6] M. Villano, A. Moreira, H. Miller, H. Rott, I. Hajnsek, R. Bamler, P. López-Dekker, T. Börner, F. De Zan, G. Krieger, K. Papathanassiou, "SIGNAL: Mission Concept and Performance Assessment,” Proc. EUSAR Conference 2010, pp. 520-523, Aachen, Germany, 08.-10. June 2010. 